\title{
Ambulatory emergency care - improvement by design
}

\author{
Authors: Mahir Mustafa Abdel Aziz Hamad ${ }^{A}$ and Vincent M Connolly ${ }^{B}$
}

\begin{abstract}
Ambulatory emergency care (AEC) has been developed by clinicians as a means of providing emergency care without the traditional bed base of a hospital. Given that AEC is provided in a clinic-style setting, it can continue to operate during periods of high bed occupancy, alleviating bed pressures and continuing to provide timely care for selected patients. Although different models of AEC have developed according to local context, there are common principles that apply to AEC services, including early access to senior decision-makers, opening hours matching demand, access to diagnostics, close collaboration with other clinical services, a mixed workforce and patient selection processes. Some of the key AEC developments have been related to technology, including high-sensitivity troponin, low-molecular-weight heparins and computer tomography (CT) pulmonary angiography. Risk stratification tools are useful for assessing the appropriateness of using AEC as a care model for patients.
\end{abstract}

\section{Introduction}

The British Association of Ambulatory Emergency Care defines ambulatory emergency care (AEC) as 'same day emergency care for patients being considered for emergency admission and early supported discharge'.1 Importantly, this care is typically provided through a clinic setting and not a bedded setting. The key clinical capacity is the staff capacity to provide healthcare. There are five broad categories of patients that can be considered for AEC:

$>$ patients to be assessed for diagnostic evaluation (eg chest pain, deep venous thrombosis etc)

> patients who require treatment provision (eg outpatient intravenous antibiotics)

> patients who require procedural intervention (eg pleural effusion management)

> patients who would benefit from input and intervention from frailty teams (eg assessment and interventions for older patients)

> patients who had been discharged early from the acute medical unit (AMU) who require an earlier review to ensure that they are continuing to improve (eg the patient with high CURB-65-

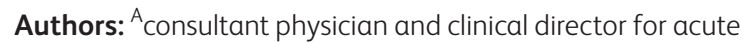
medicine, James Cook University Hospital, Middlesbrough, UK; ${ }^{B}$ regional medical director, NHS Improvement North, Quarry House, Leeds, UK scoring pneumonia or the patient with acute kidney injury who is improving but needs electrolyte rechecking).

\section{Why ambulatory emergency care?}

The traditional model of emergency care is delivered using an inpatient bed model, which was developed to provide care for patients who are sick. The profile of emergency admissions has changed considerably, with a growth in patients being admitted with ambulatory care-sensitive conditions (ACSCs) who spend fewer than 2 days in hospital, alongside a growth in older, morecomplex patients. ${ }^{2,3}$

Inpatient care has been associated with an increased risk of complications, hospital-acquired infections and harm events; therefore, the benefits and risks of admission need to be considered. ${ }^{4-7}$ Over the past 25 years, there has been a $50 \%$ reduction in hospital beds in England, accompanied by a fourfold growth in emergency admissions, which has been achieved by reducing the length of stay; however, data from winter 2017 already show high levels of bed occupancy, which could have

\section{Key points}

The design of the ambulatory emergency care (AEC) service should be driven by patient demand for services, most importantly staff capacity to provide care

Many patients currently being cared for as inpatients could have their clinical needs met in a well-designed AEC service

Patient select for $\mathrm{AEC}$ is best provided by a clinical conversation with a senior clinician often supported by using risk stratification tools

Demand for diagnostic tests should be calculated and made available to the AEC service to ensure timely reporting to inform clinical decision making

The scope of AEC services can be greatly extended through close collaboration with specialty teams to enable access to specialist opinions, services and treatments

KEYWORDS: ambulatory emergency care, acute medicine, models of ambulatory care services, patient satisfaction, the 4 Ps 
an adverse effect on patient safety and experience, and staff morale. ${ }^{8}$ A well-designed AEC can alleviate the demand for inpatient beds by reducing admissions through streaming to AEC or supporting early discharge by offering early follow-up. ${ }^{1}$

There is considerable potential for AEC services; $20 \%$ of emergency admissions were for ACSC and the rate of admission (standardising for age and sex) has grown by $26 \%$ since $2001-$ 2002. ${ }^{9}$ The NHS Institute for Innovation and Improvement estimated that one in six emergency admissions could be avoided if managed through an AEC pathway.

This conclusion was supported by The Future Hospital report, published in 2013 by the Royal College of Physicians, which recommended 'care will be organised so that ambulatory (day case) emergency care is the default position for emergency patients unless their clinical needs require admission, ${ }^{10}$

The NHS Institute for Innovation and Improvement also published the Directory of Ambulatory Emergency Care for Adults, ${ }^{11}$ which was first published in December 2007 and has been reviewed regularly. It identified 49 emergency clinical scenarios suitable for ambulatory care settings that are traditionally admitted to a hospital bed.

A similar transition to the ambulatory care model has been adopted in other countries. This has been reinforced by a recent paper that suggested that low-risk patients with a range of acute medical conditions could be treated in an outpatient setting with comparable clinical outcomes and patient satisfaction at lower costs. ${ }^{10}$ The key issue is the accurate identification of suitable patients.

\section{Patient selection}

In a properly selected cohort of patients (Box 1), AEC improves both the patient experience and the clinical outcome. ${ }^{12,13}$ Patient selection is a key step allowing the identification of patients suitable for management in the ambulatory care unit. This is best achieved when all emergency referrals are discussed with an experienced clinician, who can assess the information provided so that the patient can be streamed to the correct pathway. This decision making can be supported using a variety of presentationspecific risk stratification tools. Box 2 describes what should be available to receive patients directly. Effective implementation has been reported to reduce admissions to hospital beds by $25-30 \%$ in high-performing units, ${ }^{14}$ however, this could be difficult to implement because it depends on whether there are adequate resources and personnel as well as robust IT systems in place. ${ }^{15}$ The access to pathways is dependent on the local provision of services, which might need to be redesigned to achieve optimal use for patients.

Patient selection is essential as a risk stratification process. For defined clinical scenarios, there are several validated tools that can be used to support decision making once the diagnosis is made to facilitate early discharge. Table 1 lists some of the more common risk stratification tools.

However, for the significant number of patients for whom the initial diagnosis is not clear, a more general evaluation of the suitability of AEC for the patient is required; this includes:

$>$ assessment of the severity of illness, including the National Early Warning System (NEWS), oxygen requirements, haemodynamic stability and requirements for frequent observations

$>$ ability to maintain privacy and dignity in the AEC unit, including managing toileting needs and nutrition
Box 1. Selection of clinical diagnoses appropriate

for ambulatory care

\section{Gastroenterology}

> Upper gastrointestinal bleed with Rockall score of 0

> Lower GI bleed with no haemodynamic compromise

> Painless obstructive jaundice

$>$ Non-acute abdominal pain

$>$ Gastroenteritis

$>$ PEG related complications

\section{Endocrinology}

$>$ Hyperglycemia without ketosis

$>$ Hypoglycemia with full recovery

> Type 1 diabetes without ketosis

$>$ Electrolyte imbalances

$>$ Thyroid disease

\section{Infectious diseases}

$>$ Cellulitis

$>$ Osteomyelitis

$>$ Urinary tract infections

\section{Respiratory disease}

> Pulmonary embolism

$>$ Pneumothorax

$>$ Pleural effusions

$>$ Asthma

$>$ COPD

$>$ Community acquired pneumonia

$>$ Lower respiratory tract infections without COPD

\section{Neurology}

> Transient ischaemic attack

$>$ First seizure

> Seizure in known epileptic

$>$ Acute headache

\section{Oncology ${ }^{19}$}

Eg low risk febrile neutropaenia as determined by the MASCC score 20

\section{Miscellaneous conditions}

> Falls including syncope or collapse

$>$ Self-harm and accidental overdose

$>$ Anaemia

$\mathrm{COPD}=$ chronic obstructive pulmonary disease; $\mathrm{GI}$ = gastrointestinal; MASCC $=$ Multinational Association for Supportive Care in Cancer;

PEG = percutaneous endoscopic gastrostomy

> appropriateness for AEC staff to assess that the AEC service is best placed to meet the patient's needs and that the patient is not being moved to AEC to meet time standards or that their needs might be better served in another setting (eg transient ischaemic attack [TIA] clinic)

$>$ availability of services to meet the patient's clinical needs. 
Box 2. Potential pathways from telephone

streaming - the 6 As

Advice

Access to outpatients

Ambulatory emergency care

Assessment unit

Acute frailty unit

Admission to specialist bed

Other assessment tools, such as the ambulatory care score (AMB score), can help identify patients who are suitable for ambulatory care. The AMB score is particularly useful for less-experienced staff to guide them through an assessment. ${ }^{16}$

\section{Designing the service}

Demand and capacity

A key component of service design is understanding the true demand for the service; this includes all patients who should be treated in the AEC unit, not simply those that are treated there. Some patients whose circumstances could be managed in the AEC, might be declined or redirected to other services. Typically, the demand will be $25-30 \%$ of the acute take, but this can vary depending on the case mix. Day-to-day demand for emergency care has a natural variation, which needs to be accounted for. The availability of the service or opening hours should also take account of the periods of peak demand, which are usually late afternoon until the early evening. Once the true demand is understood, then the capacity required to deliver the service can be calculated. Capacity includes several elements, such as staffing, diagnostics and space. The capacities can be calculated for each of these elements. For example, for medical staffing, we assume that a clinical assessment by a junior doctor takes $1 \mathrm{~h}$; thus for 20 patients, $20 \mathrm{~h}$ of clinical assessment time will be required. The distribution

Table 1. Risk stratification tools to support patient streaming and early discharge

\begin{tabular}{|c|c|}
\hline Condition & Risk stratification tool \\
\hline Pulmonary embolism & $\begin{array}{l}\text { Pulmonary embolism severity } \\
\text { index (PESI), simplified PESI, } \\
\text { Hestia criteria }\end{array}$ \\
\hline $\begin{array}{l}\text { Transient ischemic attack and/or } \\
\text { cerebrovascular accident }\end{array}$ & $\mathrm{ABCD}^{2}$ and Rosier Scores \\
\hline Asthma & $\begin{array}{l}\text { Asthma severity (BTS } \\
\text { guidelines 2016) }\end{array}$ \\
\hline $\begin{array}{l}\text { Chronic obstructive pulmonary } \\
\text { disease }\end{array}$ & $\begin{array}{l}\text { BODE index and DECAF }{ }^{21} \\
\text { score }\end{array}$ \\
\hline Syncope & San Francisco Syncope Rule \\
\hline Chest pain & TIMI Score and GRACE Score \\
\hline Pneumonia & CURB score \\
\hline $\begin{array}{l}\text { Acute upper gastrointestinal } \\
\text { bleed }\end{array}$ & $\begin{array}{l}\text { Glasgow-Blatchford Bleeding } \\
\text { Score, Rockall Score }\end{array}$ \\
\hline
\end{tabular}

of demand should be taken into account with profiling of the availability of clinical staff. The mix of clinical staff is important, because senior clinical decision-makers are required to act on the outcomes of clinical and diagnostic information. A simplified capacity calculation is provided in Table 2 for illustrative purposes.

\section{Assumptions in calculating capacity}

Various assumptions are made when calculating capacity; for example (i) patients arrive at regular intervals; (ii) the junior doctor assessment can be completed in $1 \mathrm{~h}$ without interruption; and (iii) diagnostic tests are completed immediately with no delays in the availability of results. The calculation does not account for any follow-up patients. Any changes to any of these assumptions need to be factored in; for example, a 90-min timescale for completion and reporting of tests would double the required capacity.

Similarly, the availability of diagnostics should be profiled to ensure that patients have ready access to common diagnostics, such as ultrasound, computed tomography (CT) scans and echocardiography. A particularly effective use is for slots to be made available to the AEC service, which it can then fill. Using this approach minimises delays and queues, and will also ensure that patients are seen assessed and diagnosed promptly.

Appropriate nursing staffing is also important for running the service and ensuring the rapid and safe assessment of the patients in AEC; nurse staffing levels should be set to ensure that the nurse review occurs within 15 min and that appropriate times to treatment can be delivered. We recommend that the nurse:patient staffing ratio mirrors that of an emergency department. Ideally, there should be a senior sister and a nurse practitioner on every shift, in addition to staff nurses and healthcare practitioners. Approximately $30 \%$ of AEC clinical scenarios could be managed by trained nurse practitioners. ${ }^{17}$

Maintaining patient flow is a key requirement in hospitals to avoid queuing and the clinical risks associated with it. During periods of high demand, AEC units are often converted to bedded facilities overnight; this is an example of a short-term expediency worsening patient flow for days to come. The effect of bedding AEC overnight results in all potential AEC patients being admitted, resulting in higher occupancy levels, reduced flow and longer queues. It is essential that, even under pressure, the AEC unit should not be bedded; instead, the AEC service should be extended to address the problem of high demand.

\section{Training}

The close proximity of senior and junior clinical staff in the AEC unit creates an environment for teaching and training, which facilitates opportunities for direct observation and case-based discussions. Several clinical procedures occur in the AEC unit, including drainage of pleural effusions, paracentesis, lumbar puncture and insertion of nasogastric tubes, which form part of the general internal medicine (GIM) curriculum.

It is also essential that all medical staff members have the appropriate training requirements and undergo continuous regular training updates.

\section{Location}

Ideally, the ambulatory care service should be closely located to the Emergency Department (ED), with easy and rapid access 
Table 2. Sample table for calculating the required physical ambulatory emergency care (AEC) capacity ${ }^{\circ}$

\begin{tabular}{|c|c|c|c|c|c|c|}
\hline Arrival time & $\begin{array}{l}\text { No. of } \\
\text { patients }\end{array}$ & $\begin{array}{l}\text { Junior doctor } \\
\text { time (based on } 1 \\
\text { h per assessment) }\end{array}$ & $\begin{array}{l}\text { Consultant review } \\
\text { time (based on } \\
\text { 15-min review) }\end{array}$ & $\begin{array}{l}\text { Consultant follow- } \\
\text { up time (based on } \\
7.5 \mathrm{~min} \text { ) }\end{array}$ & $\begin{array}{l}\text { Trolley spaces } \\
\text { (approx. } 40 \% \text { of } \\
\text { patients using } \\
\text { trolley) }\end{array}$ & $\begin{array}{l}\text { Chair or } \\
\text { consultant room } \\
\text { (approx. } 60 \% \text { of } \\
\text { cases use chairs) }\end{array}$ \\
\hline $08.00-12.00$ & 6 & 6 & 1.5 & 0.75 & $2-3$ & $3-4$ \\
\hline $12.00-16.00$ & 10 & 10 & 2.5 & 1.25 & 4 & 6 \\
\hline $16.00-20.00$ & 4 & 4 & 1 & 0.5 & 2 & 2 \\
\hline
\end{tabular}

${ }^{a}$ The table is based on 14 new AEC patients per day, and indicates a requirement of four trolley spaces and six chairs to maintain flow during peak demand.

to diagnostics to ensure rapid transportation and assessment of patients. There should be enough waiting facilities, trolleys and consulting rooms. Where colocation with ED is not practical, consider colocation with the AMU. There are examples of newbuild AEC units that have created an environment that promotes the same-day emergency care approach with a significant degree of patient freedom to come and go between assessment, investigation and results.

\section{Patient education and experience}

Patients should be well informed, ideally at the first point of contact, about the nature of ambulatory care and the fact that they are unlikely to be admitted if they are found to be suitable for ambulatory care services (Box 3). This message can be relayed during the streaming telephone call, which can manage the expectation of patients and staff.

To continuously improve services, patients' experiences should be studied and valued. This can be in the form of 'friends and family' surveys and patient satisfaction surveys. These have been shown to be important to help develop the services in the right direction. Experience-based design is a tool that directly involves patients in the design of services, creating an environment that enhances patients' experiences. ${ }^{18}$

\section{Interface with other departments and directorate}

Effective AEC units work closely with a variety of clinical teams in the interest of patient care (Box 4 ) (eg rapid-access chest

Box 3. Advantages of developing emergency care in an ambulatory setting
> Patient acceptability
> More specialist care for patients
> Structure and predictability to the emergency process
$>$ Training opportunity
$>$ Clinical and cost effective
$>$ Alleviates bed pressures
$>$ Reduces A\&E attendances
$>$ Compliance with A\&E national 4 hour target

$\mathrm{A} \& \mathrm{E}=$ accident and emergency
Box 4. Services which can be linked to ambulatory

care

$>$ Chronic obstructive disease outreach

$>$ Pleural diseases clinics

$>$ Rapid access chest pain clinics

> Transient ischaemic attack/stroke clinics

$>$ Epilepsy clinic

> Pain management service

$>$ Functional assessment and support teams

$>$ Diabetes nurse specialist

$>$ Falls clinic

$>$ Macmillan nurses

> Outpatient parenteral antibiotics team

$>$ Home parenteral antibiotics team

$>$ Endoscopy services

Heart failure team

Mental health liaison team
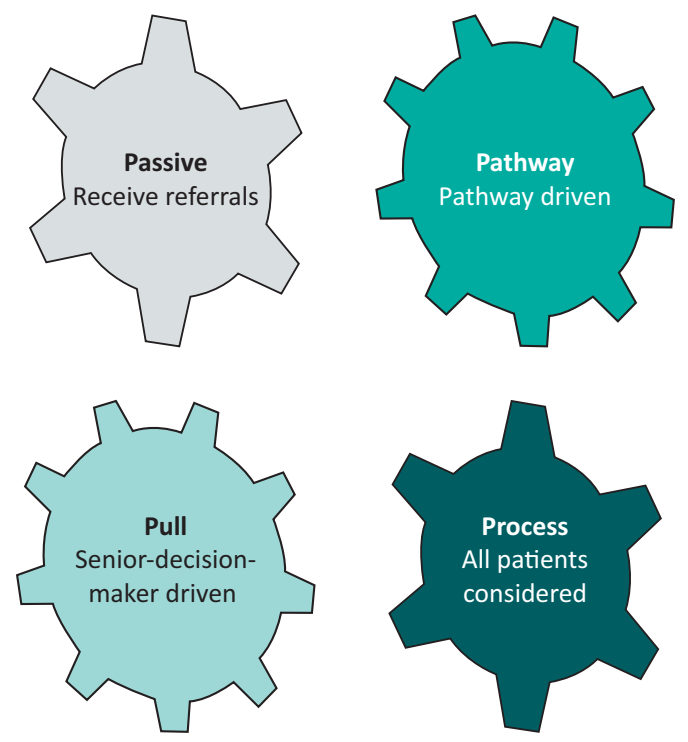

Fig 1. Models of ambulatory care - the 4 Ps. 


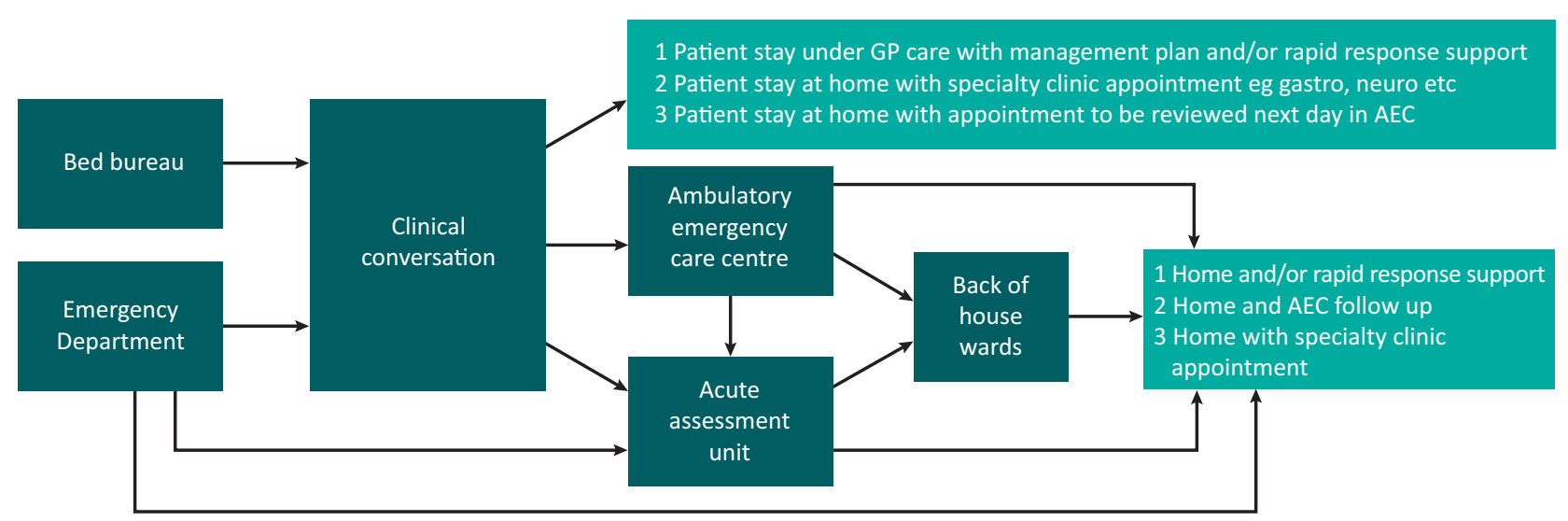

Fig 2. Example of an ambulatory emergency care (AEC) model. AEC = ambulatory emergency care

pain clinics, epilepsy clinic, pain management service, diabetes specialist nurses, Macmillan nurses, endoscopy services and self-harm services). On a day-to-day basis, each of these services might only see one or two patients, but the cumulative effect has a significant impact on patient care.

Within the AEC unit, rapid follow-up clinics and speciality clinics (gastrointestinal, chest, thromboembolic, TIA, heart failure and pleural disease clinics) can be used to manage patients being discharged from hospital, offering early access to specialty reviews, where diagnostics can be reviewed and treatment refined.

The delivery of care in the AEC setting has been enhanced by the development of outpatient pathways for intravenous antibiotics, acute respiratory illnesses and heart failure, each of which addresses the needs of specific clinical scenarios. Community-based teams, which can provide functional assessment and home care, enable AEC units to provide care for older, frail patients, who would not have previously been considered for AEC. This service is often supported by physiotherapists, occupational therapists, re-enablement specialists and social worker support, as well as access to equipment services (some trusts have equipment stores onsite). This enables the patient to be assessed quickly for mobility or care services if medically fit, ensuring that there is no delay in discharge.

\section{Models of ambulatory care services (the 4 Ps)}

There are four models of ambulatory care delivery (Fig 1).

> Passive model - where patients are admitted to AEC from the emergency department or primary care without prior consultation, discussion or having followed any clinical pathways. This is associated with low levels of patient activity and an increase in the numbers of inappropriate admissions.

> Pull model - AEC clinical staff identify suitable patients for AEC in other directorates (eg emergency department or AMU). This leads to increased levels of activity and ensures that patients are admitted appropriately to the AEC. This model is ideal when AEC services are well established, and helps to promote the service and establish $A E C$ as a mainstream process.
> Pathway-led model - this is a model in which patients are admitted and managed according to agreed clinical pathways, with inclusion and exclusion criteria. Although this model reduces variation, it can limit the scope of services by restricting the number of patients who might benefit from the service.

> Process model - this is a model in which AEC is an integral part of the emergency care system, with direct acceptance of all clinically appropriate patients based on prespecified criteria or following a clinical conversation (Fig 2).

The pathways described above can be used for high-volume clinical presentations to standardise the approach and reduce the adverse effects of variation from accepted practice. The use of pathways often simplifies access to specialist services and diagnostics using agreed criteria.

The most successful AEC models are based on the process model, with examples showing rapid improvement and positive outcomes for patients. ${ }^{18}$ This requires a senior clinician to be available to discuss referrals and with the ability to stream appropriate patients to the AEC unit.

\section{Conclusion}

Although many hospitals report that there is an AEC service available, national reporting of the use of the best-practice tariff indicates enormous levels of variation. ${ }^{18}$ Designing the $\mathrm{AEC}$ unit to meet the true patient demand will reduce these levels of variation. Clinical practice is also an important reason for variation; some units manage most cases of pulmonary embolism on an AEC unit, whereas, for others, the figure is close to zero. Sharing practice and knowledge is key to influencing clinical behaviours. Although the challenge of providing high-quality emergency care remains, an effective AEC unit goes some way to addressing this.

\section{References}

1 www.ambulatoryemergencycare.org.uk/BAAEC/About-BAAEC [Accessed 3 December 2017].

2 Blunt I. Focus on preventable admissions - trends in emergency admissions for ambulatory care sensitive conditions, 2001 to 2013. London: The Health Foundation and the Nuffield Trust, 2013. 
3 Tian I, Dixon A, Gao H. Data briefing - emergency hospital admissions for ambulatory care-sensitive conditions: identifying the potential for reductions. London: The King's Fund, 2012.

4 Barlow J, Singh D, Bayer S, Curry R. A systematic review of the benefits of home telecare for frail elderly people and those with longterm conditions. J Telemedicine Telecare 2007;13:172-9.

5 Agency for Healthcare Research and Quality. Guide to Prevention Quality Indicators: Hospital admission for ambulatory care sensitive conditions. Rockville, MD: AHRQ, 2001.

6 Bindman A, Grumbach K, Osmond D et al. Preventable hospitalizations and access to health care. JAMA 1995:274:305-11.

7 Paterson R, MacLeod DC, Thetford D et al. Prediction of in-hospital mortality and length of stay using an early warning scoring system: clinical audit, Clin Med 2006;6:281-4.

8 www.kingsfund.org.uk/projects/gp-commissioning/ten-priorities-forcommissioners/acs-conditions [Accessed 3 December 2017].

9 Blunt I. Focus on preventable admissions, Trends in emergency admissions for ambulatory care sensitive conditions, 2001 to 2013. London: QualityWatch, 2013.

10 Conley J, O'Brien CW, Leff BA, Bolen S, Zulman D. Alternative strategies to inpatient hospitalization for acute medical conditions: a systematic review. JAMA Intern Med 2016;176:1693-702.

11 Directory of Ambulatory Emergency Care for Adults, 2014. www. ambulatoryemergencycare.org.uk/BAAEC/BAAEC-Resources/ AECDirectory [Accessed 3 December 2017].

12 www.rcplondon.ac.uk/projects/outputs/future-hospital-commission [Accessed 3 December 2017].

13 UEC Review Team and ECIST. Transforming urgent and emergency care services in England, Safer, faster, better: good practice in delivering urgent and emergency care: a guide for local health and social care communities. London: NHS, 2015.

14 The Directory of Ambulatory Emergency Care for Adults, 2010. www.ambulatoryemergencycare.org.uk/BAAEC/BAAEC-Resources/ AEC-Directory [Accessed 3 December 2017].
15 LeJeune I, Sims RJA, Porter H, Mansford G, Gazis AG. What is the effect of re-introducing a clinical conversation into urgent referral pathways? Future Hosp J 2017:4:134-7.

16 Ala L, Mack J, Shaw R et al. Selecting AEC patients from the medical emergency in-take: the derivation and validation of the AMB score. Clin Med 2012:12:420-6.

17 Acute care toolkit 10, Ambulatory emergency care, 2014. www. rcplondon.ac.uk/guidelines-policy/acute-care-toolkit-10-ambulatory-emergency-care [Accessed 3 December 2017].

18 Donetto S, Tsianakas V, Roberts G. Using Experience-based Co-design (EBCD) to improve the quality of healthcare: mapping where we are now and establishing future directions. London: Kings College London, 2014.

19 Klastersky J, Paesmans M. The Multinational Association for Supportive Care in Cancer risk score: 10 years of use for identifying low risk neutropenic cancer patients. Support Care Cancer 2013;21:1487-95.

20 Cooksley T, Holland M, Klastersky J. Ambulatory outpatient management of patients with low risk febrile neutropenia. Acute Med 2015;14:178-81.

21 Steer ], Gibson J, Bourke SC. The DECAF Score: predicting hospital mortality in exacerbations of chronic obstructive pulmonary disease. Thorax 2012;67:970-76.

Address for correspondence: Dr Mahir Mustafa Abdel Aziz Hamad, James Cook University Hospital, Middlesbrough, TS4 3BW, UK.

Email:m.hamad@nhs.net 